\title{
LOS VERSOS 1050-1057 DE ELECTRA DE SÓFOCLES
}

\section{Luciano Adrián Sabattini \\ Universidad Nacional del Sur - CONICET (Argentina)}

Resumen: Este trabajo pretende tomar posición sobre la atribución de los vv. 10501051 de Electra de Sófocles a la obra fragmentaria Fedra, teniendo en cuenta la presencia y función teatrales del verbo ö $\pi \varepsilon \iota \mu$ en la tragedia griega antigua. De esta manera, sostendremos que existen motivos convincentes, aunque no suficientes, para atribuir los vv. 1050-1057 a Electra y no a Fedra.

Palabras clave: Teatro griego-Crítica textual-Tragedia fragmentaria.

\section{SOPHOCLES' ELECTRA, LINES 1050-1057}

Abstract: This paper seeks to take a position on the attribution of Sophocles' Electra 1050-1051 to the fragmentary play Phaedra, taking into consideration the theatrical presence and function of the verb ö $\pi \varepsilon \mu \mu \mathrm{in}$ ancient Greek tragedy. Thus, we will claim that there are convincing -though not sufficient- reasons to attribute lines 1050-1057 to Electra and not to Phaedra.

Keywords: Greek theatre-Textual criticism-Fragmentary tragedy.

Recibido:31.03.2017 - Aceptado: 3.05.2017

Correspondencia: Luciano Adrián Sabattini.

E-Mail: luciano.sabattini@uns.edu.ar/lucianosabattini@gmail.com Lic. en Filosofía, Ayudante de Trabajos Prácticos en Lengua y Cultura Griega I y II, Universidad Nacional del Sur (Bahía Blanca, Argentina). Becario Doctoral CONICET (Argentina). 12 de Octubre 1098, Piso 6, Gab. 13, Bahía Blanca (8000), Buenos Aires, Argentina. Teléfono celular: (+54 9) 2915774940. 


\section{Introducción}

Los versos 1050-1057 de Electra de Sófocles son objeto de fecunda discusión filológica, a raíz de que en la Antología de Juan Estobeo (3.2.29) se menciona a los vv. 1050-1051 como pertenecientes a Fedra, que ha llegado a nosotros a través de pequeños y escasos fragmentos.

En este trabajo, intentaremos tomar una posición al respecto. Primeramente, examinaremos el estado de la cuestión mediante 1) la contextualización de los vv. 1050-1057 tal como aparecen en Electra, 2) un breve análisis del estado fragmentario de Fedra y la hipotética atribución de los mencionados versos a dicha tragedia, 3) un sucinto recorrido por las posturas de la crítica filológica actual. Seguidamente, concentraremos nuestra atención en el verbo ö $\pi \varepsilon \mu \mathrm{l}(E l .1050)$ y analizaremos sus contextos de aparición en las diversas obras trágicas que se nos han conservado, con el fin de dilucidar su función teatral. De esta manera, podremos concluir que existen motivos satisfactorios para continuar ubicando los versos en cuestión en Electra, y no en Fedra.

\section{Contexto de los versos 1050-1057 de Electra de Sófocles}

La escena donde tienen lugar estos versos es el Tercer Episodio (871-1057) de la tragedia, previo al Segundo Estásimo (1058-1097). Crisótemis, hermana de Electra, aparece en escena con gran alegría porque había hallado las ofrendas de Orestes en la tumba de Agamenón, lo que daba a entender que el hermano de ambas estaba vivo y pronto a regresar a palacio, para poner fin a los abusos que Clitemnestra y Egisto cometían contra las hijas y la propiedad del otrora rey de Micenas (871-919). Electra, incrédula ante las palabras de su hermana, le refiere la llegada del pedagogo y su narración de la muerte de Orestes (920-933). Ante la situación desesperada, Electra propone a Crisótemis unirse a ella para tomar venganza contra ellos por el asesinato de Agamenón, mientras que Crisótemis le sugiere ceder ante las prerrogativas de su madre y de Egisto, ya que su condición de mujeres y el poder que detentan sus enemigos constituyen serias limitantes a su accionar (934-1014). Seguidamente, ambos personajes entran en un agón esticomítico que finaliza sin que ninguno de los dos logre convencer al otro de sus razones. Antes de retirarse del escenario y dar lugar al Segundo Estásimo, las dos hermanas (eso se cree en principio) pronuncian las siguientes líneas ${ }^{1}$ :

1 Los versos citados corresponden a la edición de Electra de Finglass (2007) y 


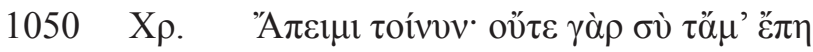

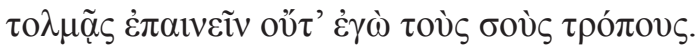

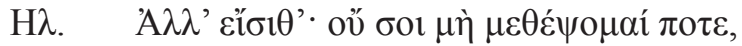

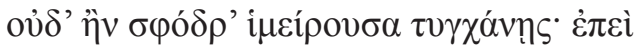

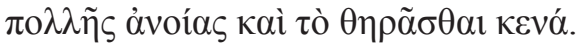

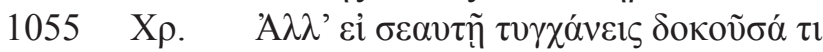

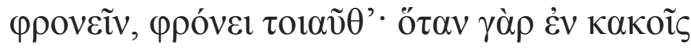

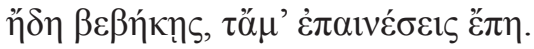

1050 Cr. Me voy entonces: pues ni tú mis palabras te resignas a aprobar, ni yo tus modos.

El. Entra ya. Nunca te seguiré, ni aunque por ventura lo desees fuertemente: ya que el perseguir metas vanas es, por lo demás, de gran insensatez.

1055 Cr. Muy bien, si acaso crees para ti misma que tienes algo de juicio, piensa eso: pero apenas te encuentres entre desgracias, aprobarás mis palabras.

La crítica filológica ha observado desde antaño el carácter problemático de los versos en cuestión. El debate en torno a ellos fue alimentado por la atribución de Juan Estobeo de los vv. 1050-1051 a Fedra de Sófocles, tragedia en estado fragmentario de la que sabemos muy poco. A continuación haremos un breve balance sobre la cantidad, condición y contexto de hallazgo de los fragmentos de Fedra de Sófocles, haciendo hincapié en la mencionada atribución. Luego, examinaremos las posturas críticas de los diversos filólogos que han tratado esta cuestión.

\section{A. Los fragmentos de Fedra de Sófocles.} Reconstrucción hipotética de su trama

Existen muy pocos fragmentos supervivientes de Fedra de Sófocles, que están compilados en diversas ediciones modernas. Algunas de las más importantes realizadas en el siglo XIX son las de Dindorf (1830), reeditada por quinta vez en 1869, Wagner (1852) y Nauck (1856), reeditada en 1889 y luego en 1964 con agregados de Bruno Snell. En los comienzos del siglo XX, destacamos la edición de Pearson (1917), que

la traducción es nuestra. 
fijó la numeración estándar de los fragmentos de Fedra. Hoy en día, las más sobresalientes son las de Radt (1977), reeditada en 1999, Lloyd-Jones (1996) y Sommerstein, Fitzpatrick y Talboy (2006). Las dos primeras siguen la numeración de Pearson, mientras que la tercera adopta una nueva. La actualmente usada para citar los fragmentos de Sófocles es la de Radt ${ }^{2}$.

Al mismo tiempo, diversos estudios filológicos, generales o particularizados, intentan rescatar la mayor cantidad de información posible de los fragmentos, así como ofrecer interpretaciones sobre su ubicación en las tragedias que se han perdido. Los más importantes son los de Bergk (1833), Welcker (1839), Carden (1974), Radt (1982), Lucas de Dios (1983) y, hoy en día, Sommerstein (2003, 2012). Mención aparte merece la edición del Hipólito de Eurípides de Barrett (1964) donde se propone un ordenamiento de los fragmentos de Fedra distinto al de Pearson.

Tabla 1. Ordenamiento de los fragmentos de Fedra según los editores (L-J = Lloyd-Jones; $\mathrm{P}=$ Page; $\mathrm{R}=$ Radt)

\begin{tabular}{|c|c|c|c|c|c|c|c|}
\hline $\begin{array}{c}\text { Wagner } \\
(1852)\end{array}$ & $\begin{array}{c}\text { Dindorf } \\
\left(1869^{5}\right)\end{array}$ & $\begin{array}{c}\text { Pearson } \\
(1917)\end{array}$ & $\begin{array}{c}\text { Nauck } \\
\left(1964^{3}\right)\end{array}$ & $\begin{array}{c}\text { Barrett } \\
(1964)\end{array}$ & $\begin{array}{c}\text { Lloyd-Jones } \\
(1996)\end{array}$ & $\begin{array}{c}\text { Radt } \\
\left(1999^{2}\right)\end{array}$ & $\begin{array}{c}\text { Sommerstein } \\
\text { et al. }(2006)\end{array}$ \\
\hline $\begin{array}{c}\text { Lyr. } A d . \\
100 \mathrm{P} \\
(P M G)\end{array}$ & $686 \mathrm{R}$ & $677 \mathrm{R}$ & $677 \mathrm{R}$ & $680 \mathrm{R}$ & $677 \mathrm{R}$ & 677 & $688 \mathrm{R}$ \\
\hline $686 \mathrm{R}$ & $677 \mathrm{R}$ & $678 \mathrm{R}$ & $678 \mathrm{R}$ & $684 \mathrm{R}$ & $678 \mathrm{R}$ & 678 & $680 \mathrm{R}$ \\
\hline $677 \mathrm{R}$ & $683 \mathrm{R}$ & $679 \mathrm{R}$ & $679 \mathrm{R}$ & $677 \mathrm{R}$ & $679 \mathrm{R}$ & 679 & 693a L-J \\
\hline $683 \mathrm{R}$ & $684 \mathrm{R}$ & $680 \mathrm{R}$ & $680 \mathrm{R}$ & $678 \mathrm{R}$ & $680 \mathrm{R}$ & 680 & $679 \mathrm{R}$ \\
\hline $684 \mathrm{R}$ & $682 \mathrm{R}$ & $681 \mathrm{R}$ & $681 \mathrm{R}$ & $679 \mathrm{R}$ & $681 \mathrm{R}$ & 681 & $684 \mathrm{R}$ \\
\hline $682 \mathrm{R}$ & $679 \mathrm{R}$ & $682 \mathrm{R}$ & $682 \mathrm{R}$ & $685 \mathrm{R}$ & $682 \mathrm{R}$ & 682 & $677 \mathrm{R}$ \\
\hline $679 \mathrm{R}$ & $681 \mathrm{R}$ & $683 \mathrm{R}$ & $683 \mathrm{R}$ & $686 \mathrm{R}$ & $683 \mathrm{R}$ & 683 & $686 \mathrm{R}$ \\
\hline $681 \mathrm{R}$ & $680 \mathrm{R}$ & $684 \mathrm{R}$ & $685 \mathrm{R}$ & $687 \mathrm{R}$ & $684 \mathrm{R}$ & 684 & $687 \mathrm{R}$ \\
\hline $680 \mathrm{R}$ & $685 \mathrm{R}$ & $685 \mathrm{R}$ & $686 \mathrm{R}$ & $683 \mathrm{R}$ & $685 \mathrm{R}$ & 685 & $687 \mathrm{a} \mathrm{R}$ \\
\hline $685 \mathrm{R}$ & $689 \mathrm{R}$ & $686 \mathrm{R}$ & $687 \mathrm{R}$ & $682 \mathrm{R}$ & $686 \mathrm{R}$ & 686 & $685 \mathrm{R}$ \\
\hline $689 \mathrm{R}$ & $688 \mathrm{R}$ & $687 \mathrm{R}$ & $688 \mathrm{R}$ & $681 \mathrm{R}$ & $687 \mathrm{R}$ & 687 & $690 \mathrm{R}$ \\
\hline $688 \mathrm{R}$ & $690 \mathrm{R}$ & $688 \mathrm{R}$ & $689 \mathrm{R}$ & $688 \mathrm{R}$ & $687 \mathrm{a}$ & $687 \mathrm{a}$ & $683 \mathrm{R}$ \\
\hline $690 \mathrm{R}$ & $678 \mathrm{R}$ & $689 \mathrm{R}$ & $690 \mathrm{R}$ & $689 \mathrm{R}$ & $\mathbf{6 9 3 a}$ & 688 & $678 \mathrm{R}$ \\
\hline
\end{tabular}

2 Para ver con algo más de detalle la historia de las ediciones de los fragmentos de Sófocles, sugerimos consultar Lucas de Dios (1983: 12-21). Un recuento más actual del estado de la cuestión es el de Sommerstein (2012: 191, n. 1). 


\begin{tabular}{|c|c|c|c|c|c|c|c|}
\hline $678 \mathrm{R}$ & $691 \mathrm{R}$ & $690 \mathrm{R}$ & $691 \mathrm{R}$ & $690 \mathrm{R}$ & & 689 & $693 \mathrm{R}$ \\
\hline $691 \mathrm{R}$ & $692 \mathrm{R}$ & $691 \mathrm{R}$ & $692 \mathrm{R}$ & $691 \mathrm{R}$ & & 690 & $682 \mathrm{R}$ \\
\hline $692 \mathrm{R}$ & $687 \mathrm{R}$ & $692 \mathrm{R}$ & $693 \mathrm{R}$ & $692 \mathrm{R}$ & & 691 & $681 \mathrm{R}$ \\
\hline $687 \mathrm{R}$ & $693 \mathrm{R}$ & $693 \mathrm{R}$ & & $693 \mathrm{R}$ & & 692 & $689 \mathrm{R}$ \\
\hline $693 \mathrm{R}$ & & & & & & 693 & $691 \mathrm{R}$ \\
\hline & & & & & & & $692 \mathrm{R}$ \\
\hline
\end{tabular}

Como vemos a partir de la Tabla 1, los fragmentos reconocidos de Fedra de Sófocles (677-693 R) son escasos, y existen serias discrepancias respecto de la atribución de determinados fragmentos a una u otra tragedia, así como de la ubicación dentro de la pieza. Según su contexto de hallazgo por parte de los editores, puede establecerse una división entre:

A. Los que entran en el conjunto de lo que Cuny llamó réflexions générales ${ }^{3}$, interesantes para un doxógrafo por tener un carácter aleccionador. Sus fuentes son:

- La Antología de Estobeo (677, 679-684, 686 R)

- La Antología de Orión (677 R)

- Los Stromata de Clemente de Alejandría $(684 \mathrm{R}=\text { Eur. } 431 \mathrm{~N})^{4}$

Los fragmentos de este grupo son los más extensos. El fr. $684 \mathrm{R}$ tiene cinco versos; es así el de mayor extensión.

B. Aquellos lexicográficamente interesantes. Sus fuentes son:

- El Lexicon de Hesiquio de Alejandría (678, 685, 687, 689-693 R)

- La Praeparatio Sophistica (Epitome) de Frínico Ático (678 R)

- La enciclopedia Suda (685 R)

- El Lexicon de Focio de Constantinopla según los Codices Berolinensis, Atheniensis (685 R) y Zavordiensis (687a R)

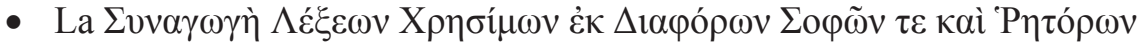

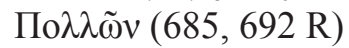

- Los Scholia Recentiora in Aristophanis Lysistratam (687a R)

- Etymologicum Magnum (688 R) ${ }^{5}$

3 Cf. Cuny (2007: 9-15).

4 Cf. Nauck (1964²: 493), Radt (1999²: 476-479).

5 Cf. Radt (1999²: 476, 479-481). 
Estos fragmentos son los de menor extensión; incluso en la mayor parte de los casos se trata de una sola palabra o dos (678, 688-693 R).

Sin embargo, algunos aparecen en antologías como en fuentes lexicográficas. El fr. $683 \mathrm{R}$ tiene como fuentes a la Antología de Estobeo y los Scholia Vetera in Lucianum ${ }^{6}$.

A partir del mito y de pocos indicios iconográficos, además de los fragmentos y de la comparación con el Hipólito conservado de Eurípides ${ }^{7}$ y la tragedia de Séneca homónima, es posible una reconstrucción, aunque muy general, de la trama de $F e d r a^{8}$. Los acontecimientos tendrían lugar en Atenas ${ }^{9}$. Seguramente los personajes intervinientes eran Fedra, Hipólito, Teseo, una nodriza ${ }^{10}$ y un coro de mujeres ${ }^{11}$. En apariencia, los temas principales tocados por Sófocles serían:

A. La pasión no correspondida de Fedra hacia Hipólito. Causada por designio divino, Fedra misma o la nodriza podría haber develado la pasión que sentía por su hijastro ${ }^{12}$. Es posible que Hipólito, antes de tomar conocimiento de esa pasión, haya hablado con un criado sobre el amor ${ }^{13}$.

6 Cf. Radt (1999²: 478).

7 Eurípides ha tratado el mito en dos ocasiones: en Hipólito Kalyptómenos (HippK, en estado fragmentario) e Hipólito Stephanóphoros (HippS, conservado en su totalidad, que sería una reacción ante las críticas contra el primero).

8 Cf. Sommerstein (2012: 198): "The material that survives from most Sophoclean tragedies is so scanty or so uninformative that we can do little more than identify the mythical episode which they dramatize -and sometimes even that is beyond us." Sin embargo, podemos reconstruir, aunque siempre teniendo en cuenta la advertencia de Lloyd-Jones (1996: 322-323), la trama de Fedra si nos basamos en Fedra de Séneca y los Scholia Vetera de Asclepíades a Odisea 11.321; cf. Radt (1999²: 475).

9 Cf. Lucas de Dios (1983: 342).

10 La presencia de la nodriza es hipotética (cf. Barrett, 1964: 37; Kiso, 1973: 25-34; Lucas de Dios, 1983: 344, n. 1361; Mills, 2003: 231), pero es bastante probable, más si atribuimos a esta obra el fr. 693a, como lo hacen Lloyd-Jones (1996: 323, 330-331) y Sommerstein, Fitzpatrick y Talboy (2006: 291).

11 S. frr. 679, 680 R. Cf. Lucas de Dios (1983: 344), Lloyd-Jones (1996: 323).

12 S. frr. 679, 680 R. Cf. Lucas de Dios (1983: 343).

13 S. fr. 684 R. Según Lucas de Dios (1983: 344, n. 1362), también es posible que 
Hipólito expondría sus razones del $\operatorname{rechazo}^{14}$ y la nodriza comunicaría a Fedra la reacción de su joven hijastro ${ }^{15}$. En unos sarcófagos romanos, según indican las apariencias, podría verse a la nodriza dándole a Hipólito una carta y a éste rechazándola ${ }^{16}$.

B. La vuelta de Teseo del Hades. Teseo volvería del Hades luego de ayudar a Piritoo ${ }^{17}$. Dado por muerto por su oîkos, sería recibido con gran sorpresa por la nodriza ${ }^{18}$ y narraría su triunfo sobre el perro Cerbero ${ }^{19}$. Este tema bien pudo haberse abordado antes del reencuentro con su esposa o después de que Teseo supiera el deseo de ésta de suicidarse, a causa del inexistente ultraje cometido por Hipólito ${ }^{20}$.

se trate de un discurso de Fedra o de la nodriza. Hay un pasaje similar en Sen. Phaed. 184-194, pronunciado por Fedra.

14 S. fr. 677 R. Para Sommerstein, Fitzpatrick y Talboy (2006: 293), este fragmento es parte del rechazo a Fedra por parte de Hipólito, antes que un descargo de Teseo en referencia a la situación. Lucas de Dios (1983: 346-347, n. 1370) no descarta ninguna de las dos opciones.

15 S. fr. 678 R. Cf. Lucas de Dios (1983: 345, 347, n. 1371). En el v. 582 de Fedra de Séneca, aparece la similar expresión 'verba sic spernit mea' en boca de la nodriza. En opinión de Sommerstein, Fitzpatrick y Talboy (2006: 297), se trata de Fedra hablando sobre el rechazo de Hipólito.

16 Cf. Kiso (1973: 28), Lucas de Dios (1983: 344, n. 1361, 345, n. 1363).

17 Cf. Barrett (1964: 12), Luque Moreno (1980: 17), Lucas de Dios (1983: 343), Sommerstein (2012: 198).

18 La nodriza se hallaba en mejores condiciones que Fedra para recibir a Teseo y entablar un diálogo. En Sen. Phaed. 835-958 hay un diálogo entre ambos que finaliza con la llegada de Teseo ante Fedra y la denuncia de ésta.

19 S. frr. 686-687a. Lucas de Dios (1983: 345, 348, nn. 1378 y 1379) conjetura que la referencia de dichos fragmentos sea el combate con Cerbero, pero Talboy (2003) lo demuestra.

20 Lucas de Dios (1983: 345) se muestra reticente a emitir una opinión, pero toma como referencia los vv. 864-958 de Fedra de Séneca. Existe la posibilidad de que Hipólito y Teseo sean los contendientes del agón, como en Hipólito Stephanóphoros, a juzgar por los frr. 677 y 683 R. 
C. El gobierno de Atenas. Contrariamente al Hipólito de Eurípides conservado hasta nuestros días ${ }^{21}$, es posible que Fedra haya incluído una cuestión política sobre el gobierno de Atenas, involucrando a Acamante y Demofonte, los hijos de Teseo y la protagonista, y la condición de hijo ilegítimo de Hipólito ${ }^{22}$. En ausencia de Teseo, Hipólito era en efecto el único en condiciones de gobernar la ciudad ${ }^{23}$. Si Teseo hubiera aludido a la posibilidad de rivalidad política con sus hermanastros (verdaderos herederos del trono pero de muy corta edad para asumirlo), en él se manifestarían las desventajas de los hijos bastardos.

El final de la tragedia es conjetural. Podemos suponer que Hipólito se habría marchado antes de la llegada de su padre (aunque es posible que padre e hijo se hayan enfrentado ${ }^{24}$ ), muriendo poco después de las maldiciones que éste le habría lanzado. Un mensajero habría narrado el suceso $^{25}$. Luego de la muerte del joven, tendría lugar el suicidio de Fedra. El Éxodo bien podría consistir en un treno final con los cadáveres de Hipólito y Fedra, si seguimos el modelo de Antígona en los finales sofocleos, a diferencia de lo que pasa en Filoctetes o en muchas obras de Eurípides, donde se empleó el deus ex machina ${ }^{26}$.

21 Scodel (2014: 231) dice respecto de Hipólito Stephanóphoros de Eurípides: "Como la tragedia desdibuja las fronteras entre el pasado distante y el presente del público ateniense, la posición de Hipólito es ambigua: mientras que los hijos de Fedra son los legítimos herederos de Teseo no está claro que la ilegitimidad de Hipólito lo pondría en las mismas desventajas en las que estaría alguien como él en la Atenas del siglo $\mathrm{V}$, y este desdibujamiento permite reacciones diversas frente al personaje".

22 Bajo el derecho de la época y no del pasado mítico recreado en la obra, Fedra debe asumir la tarea de criar a los hijos como tales. Cf. Barrett (1964: 8-9), Sommerstein (2012: 198).

23 Cf. Pearson (1917: 295), Sommerstein, Fitzpatrick y Talboy (2006: 311-312).

24 S. frr. 677 y 683 R. Barrett (1964: 12-13) dice al respecto: "It is likely, though not demonstrable, that he took no part in the action after he rejected Phaidra's advances, and perhaps that he drove straight off in horror to Trozen".

25 Siempre que nos atengamos a la tragedia homónima de Séneca. Cf. Lucas de Dios (1983: 345).

26 Cf. Lucas de Dios (1983: 346).

222 


\section{B. La atribución de la Antología de Juan Estobeo (3.2.29)}

La cita de Juan Estobeo sobre los vv. 1050-1051 de Electra ingresaría en el grupo (A) de fragmentos de Fedra, de ser esta una atribución correcta, y su presencia en Electra no sería sino una interpolación ${ }^{27}$. Concretamente, la cita de Juan Estobeo (3.2) dice:

28

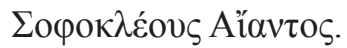

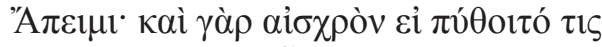

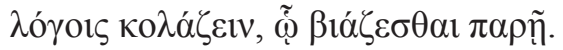

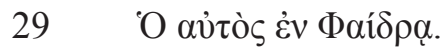

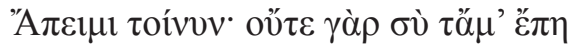

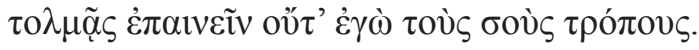

Como vimos en la Tabla 1, los editores previos a Lloyd-Jones no aceptaron la atribución a Fedra. Lloyd-Jones (1996) sí incluyó en su edición de tragedias fragmentarias los vv. 1050-1054 de Electra, ubicándolos después del fr. 687a y dándoles la numeración 693a (se omitieron los frr. 688-693 por su corta extensión) ${ }^{28}$.

La reedición en 1999 de la compilación de Radt no considera los mencionados versos como parte de Fedra. La edición de Sommerstein, Fitzpatrick y Talboy sí, aunque reordenando los fragmentos en una forma completamente distinta a Nauck, Pearson, Barrett y el propio Radt. En la Tabla 1 podemos constatar la diferencia de ubicaciones del fr. 693a entre la edición de Lloyd-Jones y la del equipo de Sommerstein. Nuestro trabajo intentará también tomar una posición en este respecto.

\section{Breve recorrido por las distintas posturas de la crítica filológica sobre la cuestión}

La crítica filológica no se ha referido al estado en que se han hallado los vv. 1050-1057 de Electra, aunque las distintas ediciones y manuscritos presentan determinadas variantes textuales.

27 Cf. Jebb (1894: LXX).

28 Lloyd-Jones (1996: 323, 330-331) tomó como base el debate que inició él junto con Wilson; cf. Lloyd-Jones y Wilson (1990: 62). 
A. Variantes textuales de los manuscritos. En el v. 1052, algunos manuscritos

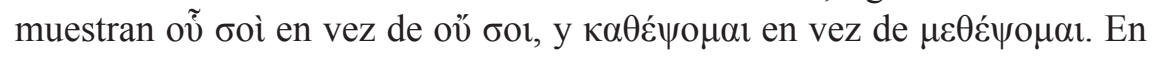

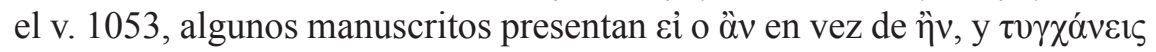

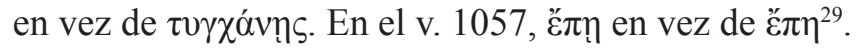

B. Variantes textuales de las ediciones. En el v. 1052, Elmsley cambia oủ $\mu$ í

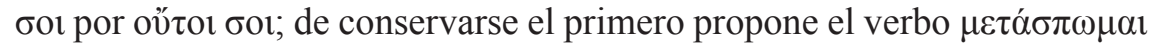

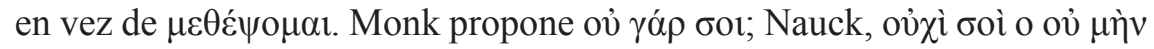

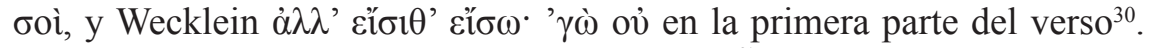
Mientras tanto, Dindorf (18764: 90) interpreta õ̃ en vez de oủ.

Constatamos así que el verso más problemático desde el punto de vista de la crítica textual ha sido el 1052. Las ediciones posteriores a la de Jebb parecen haber consensuado en la variante propuesta por este editor.

Pero las variantes textuales no son el principal problema, sino el orden de los versos, y los personajes a cargo de pronunciar las líneas en cuestión. Estos puntos, en particular, son determinantes para decidir si son efectivamente una interpolación o no.

Ya la crítica del siglo XIX advirtió las dificultades que presentan en el texto de Electra, aunque los Scholia Vetera in Electram los consideran parte de la obra ${ }^{31}$. T. Bergk propone ordenarlos así: 1055-1057, 1052-1054, 1050-105132. Por su parte, Morstadt (1864: 30-31) sostiene a) que lo dicho por Electra entre los vv. 1052-1054 es una redundancia del v. 1049; b)

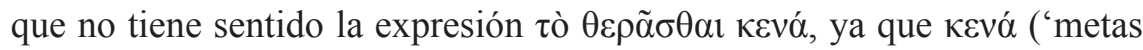
vanas') refiere a la meta de Crisótemis de que Electra logre aceptar su consejo, reconocida por ésta como un discurso sensato en el v. 1027; c) que el אaì que aparece en el v. 1054 no tiene razón de ser. Por eso, Morstadt concluye que Sófocles no escribió los vv. 1052-1054. Tampoco, se sigue de suyo, los vv. 1055-1057, porque:

Sie mutzt also ihrer Schwester auf, dass sie sich einbilde, verständig zu handeln, und fordert sie höhnisch und hähmisch auf, in dieser

29 Cf. Jebb (1894: 144), Dawe (1996³: 49), Finglass (2007: 68).

30 Cf. Jebb (1894: 144).

31 Cf. Xenis (2010: 237).

32 Cf. Jebb (1894: 144).

224 
Einbildung zu verharren. Wie kann ihr dergleichen einfallen, da Elektra nirgendwo ihr Vorhaben für ein kluges, verständiges ausgibt, sondern, im Gegentheil, ausdrücklich erklärt hat, dass es tollkühn sei und ihr den Tod bringen könne?"“ (Morstadt, 1864: 31)

Traducción: "Ella [Crisótemis] le reprocha a su hermana que imagina actuar sensatamente, y le pide sarcásticamente y con sorna que se mantenga en esta creencia. ¿Cómo puede ocurrírsele algo semejante, puesto que Electra de ningún modo hace pasar su intención por algo juicioso y sensato, sino que al contrario, ha explicado expresamente que es imprudente y que podría traerle la muerte?"

Esto es suficiente, según Morstadt, para concluir que es mejor suprimir los vv. 1052-1057, olvidando tal vez la presencia del reflexivo $\sigma \varepsilon \alpha v \tau \tilde{n}$ en el v. 1055. Sensatamente, Jebb (1894: LXXI), refiriéndose a ésta y las otras sospechas de interpolación, sostiene que:

"In a vast majority of these instances, the suspicion or rejection appears wholly unwarrantable, being due to one or more of the following causes: (1) imperfect appreciation of the censured passage in its relation to the whole context; (2) intolerance of commonplaces,- - such as 1170 and 1173; or (3) more generally, a disposition to restrict the artistic freedom of poetical and dramatic expression, by demanding that it should invariably conform (a) to rigid logic, and (b) to the verbal usages of prose." (...) "I confess that, so far as I am able to see, verse 691 is the only one in this play which affords reasonable ground for strong suspicion; and I can only hope that any students of the Electra who may consult this edition will examine each of the supposed interpolations on its own merits."

Así, tanto Jebb (1894: 144) como posteriormente Kamerbeek (1974: 141-2), suponiendo los versos como parte de Electra, han dado distintas explicaciones de las dificultades planteadas por Morstadt, sobre

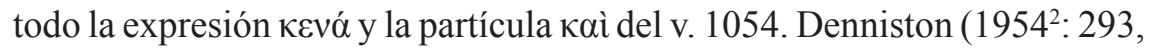
II A (2)) explica la presencia de dicha partícula en términos de "marking a minimum (descending climax)", lo que Finglass (2007: 423) consiente, pero Kamerbeek (1974: 141) impugna, proponiendo "alternatively кaì 
might be taken to mean 'actually"'33. Nosotros aceptamos esta segunda interpretación, principalmente porque aparece dentro de una cláusula

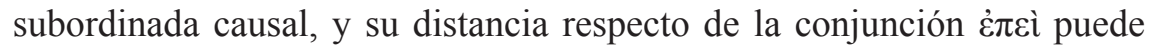
deberse a motivos métricos.

La edición de Dawe de 1996 reaviva la cuestión. Dawe (1996²: 48-49) cambia de orden los vv. 1047-1049, colocándolos en orden inverso (1049-1047), tal como lo hizo F. W. Schmidt en el siglo anterior ${ }^{34}$. Además, marca una laguna de dos versos entre 1052 y 1053, atribuyendo a Electra el v. 1052 y la primera laguna; a Crisótemis, la segunda laguna y los vv. 1053-1057.

Lloyd-Jones y Wilson (1990: 62) critican esta decisión de Dawe, diciendo que si bien las líneas 1055-57 pertenecen a Crisótemis, no serían una respuesta a los vv. 1050-1051, sino al v. 1049. Por un lado,

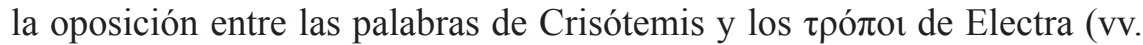
1050-1051) "seems to have very little point"; por otro, los vv. 1052-1054 no son atribuibles, por inconsistencia con el contexto, a ninguna de las dos hermanas, especialmente el v. 1054. Por ello, proponen suprimir los vv. 1050-1054, y tener en cuenta la atribución de Estobeo, ubicándolos como parte de Fedra ${ }^{35}$.

Esta posición fue sostenida en la edición de Lloyd-Jones (1996: 323, 330-331) y en Lloyd-Jones y Wilson (1997: 41). Aunque Altmeyer (2001: 208, n. 605) sostiene "Lloyd-Jones/Wilson wollen zu Unrecht die Verse 1050-1054 getilgt sehen", Zimmermann (1993: 104) aprueba los argumentos esgrimidos por los filólogos.

Siguiendo esta atribución, la edición de los fragmentos de Sófocles hecha por Sommerstein, Fitzpatrick y Talboy (2006: 276-277, 290-291, 303-305) intenta explicar la presencia de los versos en Electra, a pesar de que correspondan a Fedra, según ellos. Los editores aquí, considerando que la separación de los dos discursos es imposible, afirman:

"The fundamental problem with these five lines [i.e. vv. 10501054], as part of Electra, is that 1052-4, except for the first two words,

33 Cf. Denniston (1954²: 296-297, II B (2)).

34 Cf. Jebb (1894: 143), Dawe (1996³: 48).

35 También Finglass (2007: 422) expone, de forma más esquemática, las razones de Lloyd-Jones y Wilson. 
make no sense in Electra's mouth. It is Electra, not Chrysothemis, who, in pursuit of an aim which she "ardently... desire[s]", has asked her sister to join her in an enterprise, and been rejected; it is Chrysothemis, not Electra, who thinks the other is committing the "utter folly [of] hunt[ing] the unattainable". The passage can only be retained in Electra if these three lines can somehow be transferred to Chrysothemis; and yet that too is unsatisfactory, since it is Electra, not Chrysothemis, who remains on stage, and therefore it should be Chrysothemis who says "I'm going" (1050) and Electra who says "Yes, go" (1052)." (Sommerstein, Fitzpatrick y Talboy, 2006: 304)

No convencidos de las decisiones tomadas por Morstadt (porque en tal caso también se sacrificarían los vv. 1055-57, que no presentan problemas en ser atribuidos a Electra) y por Dawe (porque carga al personaje de Crisótemis con un discurso demasiado extenso, de seis líneas, antes de desaparecer de escena, al tiempo que infringe las convenciones de la técnica teatral normal, creando en efecto más problemas de los que resuelve), los editores concuerdan con la determinación de Lloyd-Jones: suprimen los vv. 1050-1054 de Electra y los atribuyen a Fedra. De esta manera, la aparición de dichas líneas se explica por interpolación, o bien producto de la elaboración de un actor del siglo IV a. C., o bien fruto de una coda escrita por un lector, habida cuenta de la similitud entre los vv.

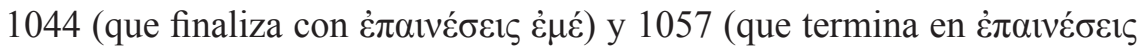
हैं $\pi)^{36}$.

Finalmente, Finglass (2007: 422-423) acepta, aunque con algunas reservas, la posición de Lloyd-Jones y Wilson: a) la oposición de ع̈ $\pi \eta$ y $\tau \rho o ́ \pi o r ~(v v . ~ 1050-1051)$ es poco elegante aunque no inaceptable; b) el v. 1052 puede atribuirse a Electra, si bien no a Crisótemis, pero la primera parte del v. 1053 es, según Finglass, "intolerably weak»; c) la cláusula que comienza con غ̇ंधì bien puede atribuirse a Crisótemis en vez de a Electra.

Actualmente, como vemos, los vv. 1055-1057 se hallan fuera de sospecha; no así los vv. 1050-1054. Desde el punto de vista de la crítica textual, el v. 1052 parece ser el más comprometido; respecto del orden y atribución de las líneas, los vv. 1052-1054 son los que presentan mayores problemas: en apariencia no son propios del discurso de ninguno de los dos personajes intervinientes. De estos, la cláusula que comienza con غ̇ं $\varepsilon \grave{~}$

36 Cf. Sommerstein, Fitzpatrick y Talboy (2006: 303-305). 


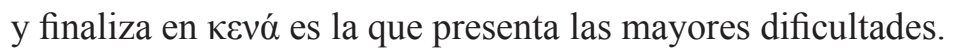

\section{El verbo ö $\pi \varepsilon \iota \mu$ en la tragedia griega}

La crítica, según hemos podido constatar, no ha tenido en cuenta en demasía la presencia del verbo ő $\pi \varepsilon \mu \mu$ ( $E l$. 1050). Este vocablo, efectivamente, se usa para marcar un "exit-speech" (Sommerstein, Fitzpatrick y Talboy, 2006: 304), un “clear exit-statement" (Finglass, 2007: 423).

Diversos editores constataron su presencia en otras tragedias del

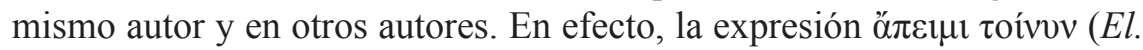
1050) es usada en dos ocasiones por Sófocles: en $O R 444$ y en el fr. 730 d.4 $\mathrm{R}^{37}$, y también por Plutarco y por Luciano ${ }^{38}$.

Finglass (2007: 423) observa también que existe un balance entre un uso del verbo ő $\pi \varepsilon 1 \mu$ y un imperativo, anterior o posterior, que ordena al interlocutor que se aleje de escena. Además de ocurrir en El. 10501052, tiene lugar en $A j$. 1159-61, donde Menelao desaparece del escenario después de discutir con el hermanastro de Áyax, Teucro. Este paralelismo se hace asimismo presente en Eurípides, en Alc. 729-30, diálogo en que el anciano Feres se aleja de escena, cumpliendo el deseo de su hijo Admeto. Los ejemplos aludidos nos permiten decir que este balance es un argumento fuerte a favor de la inseparabilidad de los dos discursos producidos en $E l$. 1050-1054.

Nos permitimos establecer la siguiente hipótesis: el uso del verbo

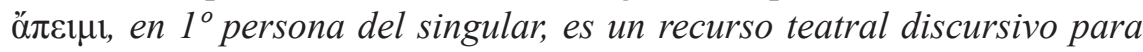
dar cuenta, al público y/o a el/los actor/es que entrará/n en la escena siguiente, no de la salida, sino de la salida definitiva de un personaje en una obra.

Constatamos esta hipótesis en las tragedias de Esquilo, Eurípides y Sófocles. En Persas de Esquilo, la sombra de Darío, antes de su desaparición, utiliza el verbo ä $\pi \varepsilon \mu 1$ en el v. 839; también Orestes en Eum. 764. Lo mismo ocurre en la obra de Eurípides, con los mensajeros en $\mathrm{Hel}$. 614 y Bacch. 1149, Feres en Alc. 730, Políxena en Hec. 414, Menelao en Andr. 733 y Antígona en Phoen. 1738. No podemos determinarlo en la fragmentaria Meleagro (fr. 27.14 Page). En una ocasión, el verbo ö $\pi \varepsilon \iota \mu$

37 Cf. Kamerbeek (1974: 141), Finglass (2007: 423). Denniston (19542: 569-570,

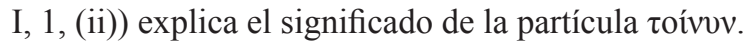

38 Cf. Finglass (2007: 423). 
es pronunciado por Meneceo, dentro de una pregunta retórica, en Phoen. 1005.

En la obra de Sófocles, son definitivas la desaparición de escena de Menelao luego de $A j .1159$ y la de Tiresias en los vv. 444 y 447 de Edipo Rey. Por desgracia, no tenemos suficientes indicios para aseverar que ocurre esto con el personaje de Teseo en el fr. 730d.4 R. Pero verificamos la hipótesis, esto es importante, con Crisótemis en El. 1050.

Encontramos excepciones a esta hipótesis, pero responden a lo que podemos interpretar como determinado tipo de efectos teatrales ${ }^{39}$. Son excepciones que, al parecer, confirman la regla. Pueden agruparse en tres tipos:

A. Reapariciones inesperadas del personaje que, líneas atrás, utilizó el verbo ó $\pi \varepsilon 1 \mu$. Esto en la obra de Sófocles tiene lugar una vez en Filoctetes: Odiseo le dice al protagonista que se marchará en el v. 124, pero reaparece en el v. 974 sorpresivamente. En la obra euripidea, podemos verlo con Hipólito en Hipp. 660, que reaparece en el v. 902, en el momento en que Teseo lo expulsa y le lanza sus maldiciones; asimismo, con Hécuba en Hec. 1054, que vuelve a escena para imprecar a Agamenón y Poliméstor. En estos tres ejemplos, el personaje reaparece para dar lugar a un virulento agón entre éste y otro/s, sea para escenificar el conflicto principal de la tragedia (como en Filoctetes e Hipólito) o para cerrar la obra (como en Hécuba).

B. Enunciaciones de desapariciones en escena que, por la intervención de otro personaje, no tienen lugar. Así, en la obra de Sófocles, el personaje de Licas, en Trach. 414, no puede irse sino hasta mucho más tarde, a pesar de sus propias intenciones. En la tragedia euripidea, otro tanto sucede con Tiresias en Phoen. 894.

C. Con negación, para indicar la condición por la que se marchará quien use el verbo. Así, por ejemplo, Creonte en Medea 275 dice que no se irá a

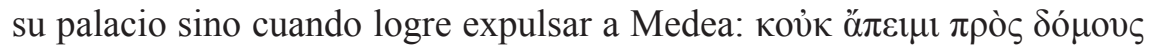

39 Esta idea se halla en relación con el hecho de que el público sabe lo que sucederá en una tragedia (porque conoce el mito que se escenifica), pero el dramaturgo selecciona episodios, perspectivas y efectos dramáticos, creando sorpresa, suspenso, etc. 


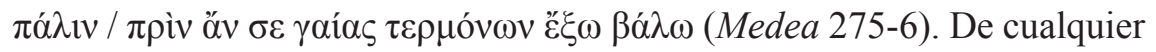
modo, es la última escena en que Creonte aparece. Lo mismo ocurre con

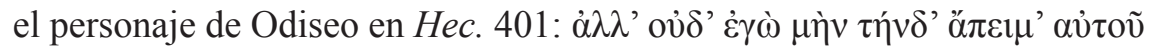
$\lambda \imath \pi \omega ́ v$. Esas, no obstante, son las últimas palabras pronunciadas por Odiseo en la obra.

Notemos, además, que solamente en una ocasión el verbo ö $\pi \varepsilon \imath \mu$ es usado por un protagonista: es el caso de Hécuba en la tragedia que lleva su nombre, que además reaparece en escena sorpresivamente.

Estamos en condiciones de afirmar que el verbo ö $\pi \varepsilon 1 \mu \mathrm{r}$ es un recurso teatral involucrado con el suspenso en la trama. De ser cierta esta hipótesis, puede tratarse de un indicio de relativa importancia para ubicar y contextualizar los fragmentos de obras dramáticas donde aparece el verbo mencionado.

\section{A favor de la atribución de los versos a Electra}

¿Cuáles serían las consecuencias de atribuir a Fedra los vv. 10501054, como lo hacen Lloyd-Jones y el equipo de Sommerstein? Prima facie, implica dar demasiado peso a la atribución de Estobeo, lo que es denunciado por Finglass (2007: 423). Aun concediendo esto, implica atribuir los versos en cuestión a al menos un personaje femenino, que es quien pronuncia las primeras dos líneas. Lloyd-Jones (1996: 323) considera que dicho personaje es nada menos que la protagonista Fedra, y su interlocutora es alguien, también mujer, cuyo consejo fue desestimado por aquella:

Sommerstein, Fitzpatrick y Talboy (2006: 276-277) desarrollan esta postura. En efecto, las líneas constituyen el intercambio final de dos personajes en un agón, una mujer (A, que pronuncia las dos primeras) y otro (B, que pronuncia las tres últimas). La mujer A le da un consejo al personaje $B$ que éste rechaza rotundamente y que posiblemente tenga que ver con una acción riesgosa. A dice que se irá, viendo que ni B aprobará sus palabras ni A su conducta. Así, B responde diciendo que nunca obedecerá a A a pesar de que lo desee, ya que perseguir una meta vana es insensato. Los editores sostienen que B no puede ser Hipólito, ya que su modo de expresar su descontento sería más vehemente. Entonces, a juzgar por el fr. 680 R, en el que alguien (Fedra) afirma que ha de sobrellevar las enfermedades divinas porque no puede escapar de las acciones vergonzosas provocadas por Zeus, cabe la posibilidad de que el personaje A sea Fedra, quien pidió 
a su nodriza que le declarara su amor por Hipólito, y B la propia nodriza, quien estaría sosteniendo la imposibilidad de convencer al joven de que se entregara a la pasión amorosa. Sabemos que luego Hipólito se enteraría del amor de Fedra a partir de la carta entregada por la nodriza, por lo que la esposa de Teseo habría intentado persuadirla una segunda vez para que lo hiciera.

Como vemos, se pone mucho peso argumentativo en explicar la

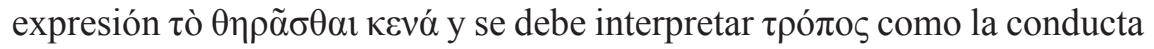
de la nodriza a tal fin.

En este punto, nuestra hipótesis sobre el uso del verbo ä $\pi \varepsilon \mu \mu$ nos proporciona un argumento válido para refutar lo sostenido por estos editores. Por supuesto, siempre que el autor no haya transgredido deliberadamente los casos de que dispusimos para establecerla.

A. Si los versos en cuestión son un diálogo de Fedra entre la protagonista y la nodriza, tendrían lugar después de un agón entre ambas, y quien utilizara el verbo ö́ $\varepsilon \varepsilon \mu$ tendría su última aparición en la obra, a menos que efectivamente sea una desaparición temporal que dé pie a un recurso teatral. Si tenemos en cuenta los paralelismos, marcados por Finglass, entre el uso del verbo ö $\pi \varepsilon \iota \mu$ y un imperativo anterior o posterior, con toda probabilidad la reaparición pudo no haberse dado, ya que así ha sucedido en los casos de Menelao en Áyax y de Feres en Alcestis de Eurípides.

Si Fedra es quien dice ö $\pi \varepsilon \varphi \mu$ y desaparece de escena, saldría nuevamente para morir, con lo que cualquier fragmento atribuible a Fedra debe ser posicionado antes del 693a L-J. Los sucesos posteriores referidos a Fedra serían narrados por un mensajero o por la nodriza, no pudiendo reencontrarse aquella viva ni con Teseo ni Hipólito. Pero la posibilidad de que se trate de Fedra se reduce si observamos que sólo en una ocasión un protagonista usa este verbo, y en esa ocasión no es acompañado por un imperativo anterior o posterior dicho por un interlocutor. Sólo la transgresión de estas convenciones (que nuestra hipótesis ha establecido como tales), posibilitaría que Fedra apareciera sorpresivamente en una escena previa a su muerte. Esto resulta poco convincente, porque los dos primeros versos del fragmento denotan una frialdad inusual en un suicida. Comparemos lo que ocurre por ejemplo con Áyax en $\mathrm{Aj}$. 646-692, donde el protagonista da un discurso previo a su muerte usando dos veces un verbo $\varepsilon \tilde{u} \mu \mathrm{y}$ no ö́ $\varepsilon \uparrow \mu \mathrm{l}$, dando a entender que volverá a aparecer, aunque para 
eliminarse, en los vv. 654 y 690.

Si es la nodriza quien dice ő́ $\varepsilon \varepsilon \mu 1$ y desaparece de escena, ya no tendría lugar el acercamiento a Hipólito ni el diálogo con Teseo, y no cabría la posibilidad de una reaparición sorpresiva del personaje (¿con qué fin?). Los versos deben tener lugar después de cualquier fragmento atribuible a la nodriza, pero en ese caso, no es posible que ella, después del agón con Fedra, dialogue con Teseo. Existe la posibilidad de que el interlocutor de

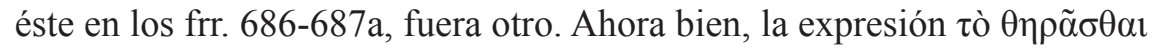

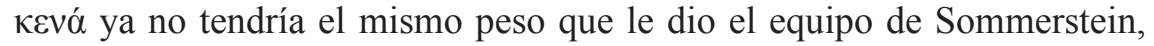
quienes advirtieron que no parecía tener sentido que la nodriza haya pronunciado los versos de salida de escena. Por lo tanto, nos parece discutible la atribución de $E l$. 1050-1 a cualquiera de estos dos personajes, como mínimo.

B. Aun pensando que el fragmento en cuestión fuera parte de Fedra, lo cual no parece ser el caso, es tan arriesgado situar este fragmento al inicio de la obra como hacerlo al final, porque es claro que el personaje A es una mujer, y más posiblemente secundaria que protagonista. Como mínimo, los discursos tienen lugar al final del Primer Episodio; como máximo, antes de la vuelta de Teseo. Por ende, no coincidimos con el ordenamiento del fr. 693a propuesto por Sommerstein, Fitzpatrick y Talboy (2006: 291).

C. Si el fragmento perteneciera a Fedra, aportaría en efecto nuevos elementos interesantes a la reconstrucción de la tragedia, y esto es posible sólo en tanto el dramaturgo transgreda las técnicas dramáticas conocidas planteadas por nuestra hipótesis, que no habituales, porque no debemos olvidar que tenemos una enorme porción de tragedias perdidas.

Si seguimos la hipótesis, ciertamente, resulta mucho más seguro considerar el fragmento en cuestión como parte de Electra, y no de Fedra. Ahora bien, esto implica aceptar la acertada observación de Finglass:

"The interpolation may have been motivated by a Desire to lengthen the stichomythia by supplying a clear exit-statement for Chrysothemis. (...) These weak lines are probably better regarded as an actor's interpolation than the work of Sophocles." (Finglass, 2007: 423). 
Se trata de una inserción ${ }^{40}$ de un actor, perteneciente a Electra, no escrita por Sófocles. Según nuestra hipótesis, los versos se adecuan a la última aparición de Crisótemis en escena, y es posible ver claramente por qué los versos fueron colocados allí: para dar clara cuenta de su salida de escena.

\section{Conclusiones}

Teniendo en cuenta la presencia y función teatrales del verbo $\ddot{\alpha} \pi \varepsilon \mu \mu \mathrm{r}$ en la tragedia griega antigua, de ser correctas nuestras apreciaciones, hay motivos convincentes, aunque no suficientes (ya que siempre cabe la posibilidad de que el dramaturgo decida transgredir las convenciones teatrales; convenciones que, por otro lado, son planteadas en nuestra hipótesis bajo la evidencia de un corpus extremadamente limitado), para atribuir los vv. 1050-1057 a Electra y no a Fedra.

\section{Referencias bibliográficas}

ALTMEYER, M. (2001). Unzeitgemäßes Denken bei Sophokles. Stuttgart: Franz Steiner Verlag.

BARRETT, W. S. ed. (1964). Euripides: Hippolytos. Oxford: Oxford University Press.

BERGK, T. (1833). Commentatio de Sophoclis fragmentis. Leipzig: Staritz.

CARDEN, R. (1974). The Papyrus Fragments of Sophocles. Berlin: De Gruyter.

CUNY, D. (2007). Une leçon de vie: Les réflexions générales dans le théâtre de

Sophocle. Paris: Les Belles Lettres.

DAWE, R. D. ed. $\left(1996^{3}\right)$. Sophocles: Electra. Stuttgart: Springer Fachmedien Wiesbaden.

DENNISTON, J. D. (1954²). The Greek Particles. Oxford: Clarendon Press.

DINDORF, W. ed. $\left(1869^{5}\right)$. Poetarum Scenicorum Graecorum. Leipzig: B. G. Teubner.

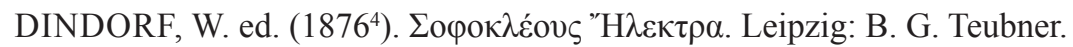

FINGLASS, P. J. ed. (2007). Sophocles: Electra. Cambridge: Cambridge University Press.

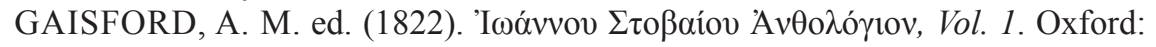
Clarendon Press.

HAMILTON, R. (1974). “Objective Evidence for Actors' Interpolations in Greek Tragedy", Greek, Roman and Byzantine Studies, 15, 4, 387-402.

40 La inserción es una forma de interpolación. Cf. Hamilton (1974: 387, n. 1) para una definición de interpolación y los estudios realizados hasta 1974. 
JEBB, R. C. ed. (1894). Sophocles: The Plays and Fragments, Part VI: The Electra. Cambridge: Cambridge University Press.

KAMERBEEK, J. C. (1974). The Plays of Sophocles, Part V: The Electra (Commentaries) Leiden: Brill.

KISO, A. (1973). "Sophocles' Phaedra and the Phaedra of the first Hippolytus", Bulletin of the Institute of Classical Studies, 20, 1, 22-36.

LUCAS DE DIOS, J. M. (1983). Sófocles: Fragmentos. Madrid: Gredos.

LUQUE MORENO, J. ed. (1980). Séneca: Tragedias II. Madrid: Gredos.

LLOYD-JONES, H. ed. (1996). Sophocles, Vol. 3: Fragments. Massachusetts: Harvard University Press.

LLOYD-JONES, H. y WILSON, N. G. (1990). Sophoclea: Studies on the Text of Sophocles. Oxford: Oxford University Press.

LLOYD-JONES, H. y WILSON, N. G. (1997). Hypomnemata: Sophocles Second Thoughts. Göttingen: Vandenhoeck \& Ruprecht.

MILLER, F. J. ed. (1938). Seneca: Tragedies Vol. 1. London: Heinemann.

MILLS, S. (2003). "Sophocles' Aegeus and Phaedra", Sommerstein, A. ed. (2003). Shards from Kolonos: Studies in Sophoclean Fragments. Bari: Levante Editori, 219-232.

MORSTADT, R. (1864). Beiträge zur Exegese und Kritik der sophokleischen Tragödien Elektra, Aias und Antigone. Schaffhausen: Hurter'sche Buchhandlung.

NAUCK, A. ed. (1964³). Tragicorum Graecorum Fragmenta. Hildesheim: Georg Olms Verlagsbuchhandlung.

PAGE, D. L. ed. (1941). Select Papyri, Vol. 3: Literary papyri. London: Heinemann.

PAGE, D. L. ed. (1962). Poetae Melici Graeci. Oxford: Clarendon Press.

PEARSON, A. C. ed. (1917). The Fragments of Sophocles, Vol. II. Cambridge: Cambridge University Press.

RADT, S. (1982). "Sophokles in seinen Fragmenten", Entretiens Hardt, 29 (Sophocle), 185-231.

RADT, S. ED. (19992). Tragicorum Graecorum Fragmenta, Vol. 4: Sophocles. Göttingen: Vandenhoeck \& Ruprecht.

SCODEL, R. (2014). La tragedia griega: una introducción (Barreiro, E. J. trad.). México D. F.: FCE.

SMYTH, H. W. ed. (1922-1926). Aeschylus in Two Volumes. London: Heinemann.

SOMMERSTEIN, A. (2012). "Fragments and Lost Tragedies", Markantonatos, A. ed. (2012). Brill's Companion to Sophocles. Leiden: Brill, 191-209.

SOMMERSTEIN, A. ed. (2003). Shards from Kolonos: Studies in Sophoclean Fragments. Bari: Levante Editori.

SOMMERSTEIN, A., Fitzpatrick, D., Talboy, T. eds. (2006). Sophocles: Selected Fragmentary Plays, Vol. I. Oxford: Aris \& Philips. 
STORR, F. ed. (1912-1919). Sophocles in Two Volumes. London: Heinemann.

TALBOY, T. (2003). "A Tell-Tale Tail: Sophocles Phaidra fr. 687 and 687a", Sommerstein, A. ed. (2003). Shards from Kolonos: Studies in Sophoclean Fragments. Bari: Levante Editori, 233-240.

WAGNER, F. W. ed. (1852). Poetarum Tragicorum Graecorum Fragmenta, Vol. 1. Bratislava: Trewendt \& Granier.

WAY, A. S. ed. (1930-1946). Euripides in Four Volumes. London: Heinemann.

WELCKER, F. E. (1839). Die Griechischen Tragödien. Bonn: Eduard Weber.

XENIS, G. A. ed. (2010). Scholia Vetera in Sophoclis "Electram". Berlin: De Gruyter.

ZIMMERMANN, B. (1993). "H. Lloyd Jones et N. G. Wilson. Sophoclis fahulae \& H.Lloyd-Jones and N. G. Wilson: Sophoclea” (reseña), Gnomon, 65, 2, 100-109. 